\title{
Estudio de la estructura y las propiedades mecánicas en un recubrimiento de circona estabilizada con $8 \%$ en mol de itria elaborado por proyección térmica por plasma a partir de suspensiones
}

\author{
Study of the structure and mechanical \\ properties in $8 \mathrm{~mol} \%$ yttria-stabilized \\ zirconia coating manufactured by \\ suspension plasma spraying
}

Francy Milena Hurtado ${ }^{1}$, Andrés González Hernández ${ }^{1,2}$, María Esperanza López Gómez ${ }^{1}$, Hélène Ageorges²

\footnotetext{
${ }^{1}$ Departamento de Ingeniería de Materiales, Grupos GIPIMME-GIMACYR, Universidad de Antioquia, Medellín, Colombia. e-mail: francymhurtado@gmail.com; esperanza.lopez@udea.edu.co .

2 SPCTS, UMR CNRS 6638, Université de Limoges, Faculté des Sciences et Techniques, 123 Avenue Albert Thomas, 87060 Limoges, France.e-mail: andresggonzalezh@gmail.com, helene.ageorges@unilim.fr
}

\section{RESUMEN}

El estudio de recubrimientos nanoestructurados fabricados mediante proyección térmica ha generado un gran interés en las últimas dos décadas debido a su considerable mejora en sus propiedades sobre todo en las propiedades físicas y mecánicas. Esta mejora resulta de reducir los tamaños de grano de su materia prima a escalas de 100 a 1000 veces más bajas comparadas con los recubrimientos convencionales microestructurados. Así, estos recubrimientos con estructuras a nivel nano y/o submicrométrica están empezando a ser usados en la industria gracias a sus sobresalientes propiedades y es fundamental el estudio de sus propiedades mecánicas para determinadas aplicaciones. En la actualidad, la indentación es la principal técnica de caracterización de recubrimientos y películas delgadas, a pesar de estar altamente influenciada por los defectos propios del recubrimiento en la vecindad de la indentación. La nanoindentación y la microindentación se presentan como la mejor opción para evaluar el comportamiento mecánico de estos recubrimientos nanoestructurados. Por esta razón, en este trabajo se analizó el efecto de la estructura de un recubrimiento de circona estabilizada con $8 \%$ en mol de itria (8YSZ) elaborado por proyección térmica por plasma a partir de suspensiones nanométricas (SPS) y su relación con sus propiedades mecánicas (dureza y módulo de elasticidad) medidas por las técnicas de nanoindentación y microindentación. El análisis de la estructura de la sección transversal mostró que el recubrimiento exhibe una estructura bimodal, la cual está compuesta por una zona con partículas nanométricas semifundidas (ZS) y lamelas con mayor grado de fusión (ZF). Los ensayos de nanoindentación mostraron una distribución de Weibull bimodal de sus propiedades mecánicas, la cual característica de este tipo de recubrimientos; mientras que la dureza (HV) y el módulo de elasticidad evaluados por microindentación, presentaron una distribución monomodal. Estos resultados de microindentación estuvieron influenciados por el área de contacto en las zonas indentadas en la estructura bimodal del recubrimiento.

Palabras clave: Recubrimiento por plasma a partir de suspensiones (SPS), estructura, indentación, Distribución Weibull, recubrimiento 8YSZ.

\section{ABSTRACT}

The study of nanostructured coatings manufactured by thermal spraying has generated a great interest in the last two decades due to their considerable improved properties especially in the physical and mechanical properties. This improvement is to reduce grain sizes of their raw materials at scales of 100-1000 times lower compared to conventional coatings (microstructured). Thus, these coatings structured at the nano and sub micrometric scale start to be used in the industrial applications and, it is essential to study their mechanical properties for certain applications. Currently, the indentation is the main technique for characterizing thin films and coatings, despite being highly influenced by the defects of the coating in the vicinity of the indentation. Nanoindentation and microindentation is presented as the best option for evaluate the mechanical be- 
havior of nanostructured coatings. For this reason, in this work the study of the effect of the structure of the 8 mol\% yttria-stabilised zirconia coating (8YSZ) manufactured by suspension plasma spraying (SPS) and the relationship with its mechanical properties (hardness and elastic modulus) measures for nanoindentation and microindentation techniques was analyzed. Analysis of the structure of the cross section of coating exhibit a bimodal structure, which is composed by a zone with semi-molten nanoparticles (ZS) and lamellaes with a higher grade of fusion (ZF). Nanoindentation tests showed a bimodal Weibull distribution of the mechanical properties (hardness and elastic modulus) which is related to the two zones (ZF and ZS) present in the coating, while hardness (HV) and elastic modulus measured by microhardness, showed a monomodal distribution. These results of microhardness were influenced by the contact area of the indentation in zones composed of the bimodal structure of the YSZ coating.

Keywords: Suspension plasma spraying (SPS), structure, indentation, Weibull distribution, 8YSZ coating.

\section{INTRODUCCIÓN}

Los recubrimientos de circona estabilizadas con itria (conocida en ingles por sus siglas: YSZ - Yttria Stabilised Zirconia) son usados en una gran variedad de aplicaciones estructurales y funcionales gracias a su buenas propiedades mecánicas en especial a alta temperatura. En particular, estos recubrimientos son usados como una capa superior en sistemas que actúan como barreras térmicas gracias a su alta estabilidad química, baja conductividad térmica y alta tenacidad [1-5]. También, los recubrimientos de YSZ son utilizados en pilas de combustible de óxido sólido (conocido en inglés como SOFC), los cuales son dispositivos electroquímicos que convierten la energía química en eléctrica y en especial la capa de YSZ funciona como electrolito [6].

Una de las técnicas más utilizadas para obtener estos recubrimientos es la proyección térmica por plasma conocida como APS (Atmospheric Plasma Spraying), la cual consiste en inyectar polvos micrométricos al plasma donde son fundidos o semifundidos y luego proyectados hasta que impactan el sustrato. Estas partículas fundidas o semifundidas forman un apilamiento a una rápida velocidad de enfriamiento, de tal manera que forman individuales laminillas o lamelas obteniendo así el recubrimiento [1,7].

Por otro lado, desde el comienzo de la década de los 90's la comunidad científica ha empezado a investigar el uso de la proyección térmica para obtener recubrimientos nanoestructurados [8]. El interés por obtener estos recubrimientos que retengan en su estructura zonas nanométricas se debe a que este tipo de estructura mejoran las propiedades mecánicas de los recubrimientos con respecto a los recubrimientos microestructurados, especialmente la tenacidad a la fractura, ya que inhibe el crecimiento y la propagación de las grietas [9]. Actualmente hay dos formas de obtener recubrimientos nanoestructurados: (i) mediante proyección térmica de partículas nanométricas aglomeradas, ó (ii) mediante la proyección térmica a partir de suspensiones de partículas nanométricas conocida como SPS (por sus siglas en ingles Suspension Plasma Spraying).

La técnica de SPS difiere de la técnica de APS principalmente porque utiliza un líquido portador de las partículas nanométricas (suspensión) en lugar de un gas de arrastre del polvo micrométrico [10]. Este cambio soluciona los problemas cuando se inyecta polvos nanométricos, ya que debido a su bajo flujo másico generan obstrucciones de los sistemas de alimentación. De esta forma, la técnica SPS permite proyectar tamaños de partícula submicrométrico y nanométrico, lo cual implica cierta ventaja con respecto a las técnicas convencionales, las cuales están limitadas únicamente a tamaños micrométricos [11]. En los recubrimientos elaborados por SPS, su materia prima es una suspensión que contiene partículas nanométricas donde su aglomeración es controlada por acción mecánica (agitadores) o/y por acción química (mediante dispersantes o defloculantes) [12]. Luego, la suspensión es inyectada preferiblemente a la zona de alta temperatura del jet de plasma, donde se desintegra y forma pequeñas gotas que se evaporan dejando que las partículas nanométricas queden en contacto con el plasma para luego fundirse o semifundirse y así ser proyectadas para formar el recubrimiento.

De otro lado, muchos trabajos de investigación han sido publicados en los últimos años sobre la caracterización de las propiedades mecánicas de materiales y recubrimientos cerámicos mediante el uso de la técnica de nanoindentación. Sin embargo, la cantidad de artículos que tratan el estudio de la técnica de nanoindentación para caracterizar capas de SPS es muy escasa [3-6,8]. De los más importantes, Wang et al. [13] y Pawłowski et al. [14] han investigado la estructura y las propiedades mecánicas por indentación de un recubrimiento de YSZ obtenido por SPS a partir de un polvo nanoestructurado. En ambos estudios los recubrimientos mostraron una estructura bimodal formada por partículas nanométricas rodeadas por lamelas completamente fundidas, lo que causó una distribución bimodal de las propiedades (dureza y módulo de elasticidad). Otro trabajo como el de Carpio et al. [15], estudió la influencia de las materias primas en suspensión acuosa con una distribución de tamaño de partícula submicrométrica y nanométrica en las propiedades mecá- 
nicas de recubrimientos de YSZ elaborados por SPS. Así encontró que mediante nanoindentación, todos los recubrimientos muestran una estructura formadas por zonas parcialmente fundidas que contienen partículas de tamaño submicrométrico rodeadas de zonas completamente fundidas. Estas áreas parcialmente fundidas aumentaron altamente con la distancia de proyección y se estableció una clara correlación entre las propiedades mecánicas y la cantidad de zonas parcialmente fundidas.

Por otra parte, durante el proceso de proyección térmica se generan inevitablemente micro-defectos o características estructurales que influyen en las propiedades mecánicas de los recubrimientos. Debido a esto, es importante determinar el tamaño y forma de los micro-defectos para elegir la técnica de caracterización mecánica más adecuada. En la actualidad, la indentación es la principal técnica de caracterización de recubrimientos y películas delgadas, a pesar de estar altamente influenciada por los defectos propios del recubrimiento en la vecindad de la indentación, que con un buen tratamiento estadístico puede generar resultados representativos. La nanoindentación y microindentación se presentan como la mejor opción para evaluar el comportamiento mecánico de recubrimientos nanoestructurados, ya que la nanoindentación, al aplicar cargas del orden de los $\mathrm{mN}$ y por la puntiaguda forma del indentador (Berkovich) permite realizar pequeñas indentaciones que distinguen entre las propiedades mecánicas de cada región característica de recubrimientos nanoestructurados, en especial los elaborados por proyección térmica. La microindentación, por otro lado, permite aplicar cargas del orden de los $\mathrm{N}$ y realizar así una evaluación más global de las propiedades mecánicas de un recubrimiento.

En este artículo se hace un análisis del efecto de las diferentes regiones estructurales que conforman un recubrimiento de $8 \mathrm{YSZ}$ elaborado por SPS con respecto a sus propiedades mecánicas medidas por las técnicas nanoindentación y microindentación.

\section{METODOLOGÍA EXPERIMENTAL}

En este trabajo se realizó una caracterización morfológica de la materia prima con la que se elaboraron los recubrimientos, luego se preparó una suspensión con dichas partículas y después se utilizó la técnica de SPS para elaborar el recubrimiento de 8YSZ. En este recubrimiento se analizó el efecto de la estructura en las propiedades mecánicas evaluadas mediante nanoindentación y microindentación. A continuación se describen todas las técnicas utilizadas en este estudio.

\subsection{Caracterización morfológica de la materia prima para elaborar los recubrimientos}

La caracterización morfológica del polvo nanométrico 8YSZ utilizado como materia prima para elaborar la suspensión fue realizada mediante un microscopio electrónico de barrido de emisión de campo (FE-SEM: Field Emission Scanning Electron Microscopy) de marca JEOL 7400F. El polvo nanométrico de composición 8 YSZ fue fabricado por la casa comercial US Research Nanomaterials.

\subsection{Elaboración del recubrimiento}

Para elaborar el recubrimiento por proyección térmica por plasma a partir de suspensiones, se utilizó una antorcha convencional monocatódica tipo F4-MB (Wolhen, Suiza) con el sistema Multicoat de Sulzer Metco® y un sistema de inyección de la suspensión, el cual estaba montado en el laboratorio SPCTS de la Universidad de Limoges (Francia). Este sistema consta de dos recipientes presurizados donde en uno se almacena la suspensión previamente preparada y en el otro se almacena etanol para limpiar el sistema después de cada ensayo. El inyector tenía un diámetro de $150 \mu \mathrm{m}$ y es ajustado manualmente de manera que la fragmentación de la suspensión se llevara a cabo dentro del jet de plasma. La suspensión fue preparada con etanol como solvente y un dispersante "Beycostat C213" el cual fue utilizado para evitar la aglomeración de las partículas nanométricas. La suspensión de 8YSZ utilizada en este estudio ha sido optimizada por EtchartSalas [16] y Bacciochini [17], la cual tiene un porcentaje de sólidos del $20 \%$ con un cantidad de dispersante del $2.1 \%$ de la masa del polvo. En primera instancia, el dispersante fue diluido en etanol mediante agitación magnética y con una sonda ultrasónica de alta potencia. Luego, el polvo fue adicionado en pequeñas cantidades para evitar la formación de grumos. El material de los sustratos utilizados para la elaboración del recubrimiento fue acero inoxidable del tipo AISI/SAE 304L con una diámetro de $25.4 \mathrm{~mm}$ y espesor de $9 \mathrm{~mm}$. Este tipo de sustratos generalmente es utilizado en procesos industriales a alta temperatura y equipos expuestos a ambientes corrosivos. Estos fueron pulidos hasta lograr un acabado espejo.

Ensayos preliminares realizados permitieron definir los parámetros de elaboración de los recubrimientos por SPS. Para el proceso se establecieron los siguientes parámetros: una distancia entre la punta de la 
antorcha y el sustrato de $3 \mathrm{~cm}$; una intensidad de corriente en la antorcha de $500 \mathrm{~A}$; gases plasmagénicos con una relación de Ar-He con un flujo de 40 - 20 slpm respectivamente; una temperatura de precalentamiento del sustrato $200^{\circ} \mathrm{C}$ y un tiempo de aplicación de 4 min sin superar los $400^{\circ} \mathrm{C}$.

\subsection{Caracterización morfológica, química y mecánica del recubrimiento}

La muestra del recubrimiento de 8YSZ obtenido por SPS para la caracterización morfológica y mecánica, fue pulida teniendo en cuenta la norma ASTM E1920-03 [18]. Antes de ser cortada, la muestra fue montada en resina (Buehler EpoxiCure ${ }^{\circledR}$ o Epothin ${ }^{\circledR}$ ) y fue dejada endureciéndose durante aprox. 24 h. Después del proceso de corte en compresión del recubrimiento, se realizó un desbaste con papel lija de $\mathrm{SiC}$ de diferentes granos con una secuencia de P320, P600, P1000, P2000 y P4000. Por último, se realizó un pulido con una pasta de diamante de $1 \mu \mathrm{m}$ y su lubricante líquido. La velocidad de rotación en la máquina de pulido fue entre 200-300 rpm acorde a lo estipulado en la norma ASTM E1920-03 [18]. El análisis de la sección transversal pulida del recubrimiento y la morfología de las indentaciones producto de los ensayos de microindentación y nanoindentación fueron analizadas mediante un microscopio de electrónica de la marca JEOL JSM6490LV. Para verificar la composición química del recubrimiento se utilizó la técnica de fluorescencia de rayos X (FRX) por longitud de onda dispersiva, utilizando un espectrómetro de OPTIM'X de Thermo Scientific. En cuanto a la caracterización mecánica del recubrimiento, a continuación se describen las técnicas de nanoindentación y microindentación utilizadas en este trabajo.

\subsubsection{Nanoindentación}

Los ensayos de nanoindentación se realizaron en un equipo Nano Indenter XP de MTS Systems Corporation. Se hicieron 12 indentaciones en línea recta espaciadas $40 \mu \mathrm{m}$ cada una, realizadas en la mitad de la sección transversal pulida del recubrimiento, evitando así el efecto del sustrato y el borde del recubrimiento. Las indentaciones Berkovich fueron distribuidas en tres regiones a lo largo de la sección transversal. Las cargas estudiadas preliminarmente estuvieron en un rango entre 100 a $500 \mathrm{mN}$. De este rango de cargas se seleccionó experimentalmente una carga de $100 \mathrm{mN}(10 \mathrm{~g})$ para realizar las medidas de dureza y módulo de elasticidad, utilizando el modo continuos stiffness measurement (CSM) que permite obtener una medida continúa de los datos. La dureza (H) y el módulo de elasticidad (E) se calcularon a partir de los datos de la curva cargadescarga P-h obtenida con un indentador Berkovich mediante el método de Oliver y Pharr [19]. Esta dureza y módulo de elasticidad era calculada directamente por el software del equipo.

\subsubsection{Microindentación}

Los ensayos de microindentación se realizaron en un equipo Shimadzu serie G20 y se hicieron bajo la norma ASTM C1327-08 [20] para indentaciones Vickers y bajo la norma ASTM C1326-03 [21] para indentaciones Knoop [21]. Se utilizó un indentador Vickers para calcular la dureza Vickers (HV) variando la carga entre 25 y $100 \mathrm{~g}$ y se hicieron 20 medidas por cada carga en la sección transversal pulida del recubrimiento. Esto se hizo para encontrar la carga crítica a la cual la recuperación elástica no es tan pequeña ni tan grande, que se considera que no hay una sobreestimación ni una subestimación de la microdureza. Se encontró una carga crítica de $60 \mathrm{~g}$ para el recubrimiento estudiado, la cual se aplicó durante $15 \mathrm{~s}$ espaciadas $40 \mu \mathrm{m}$ una de la otra, para evitar la influencia de las indentaciones vecinas. Se seleccionaron 13 huellas aceptables por la norma de las 20 realizadas.

Por otro lado, se utilizó un indentador Knoop para hallar la dureza Knoop (HK), la cual fue utilizada para calcular el módulo de elasticidad (E), utilizando el método de Marshall. Para el ensayo se hicieron 20 indentaciones Knoop a una carga de $125 \mathrm{~g}$. La carga fue aplicada durante $15 \mathrm{~s}$ y las huellas fueron distribuidas en toda la muestra a una distancia de aproximadamente $150 \mu \mathrm{m}$ una de la otra, para evitar la influencia de las indentaciones vecinas. Se seleccionaron 13 huellas aceptables por la norma de las 20 realizadas en la sección transversal del recubrimiento.

\subsubsection{Distribución de Weibull}

Los datos del módulo de elasticidad y dureza, hallados por las dos técnicas de indentación, fueron analizados mediante la distribución estadística de Weibull. Esta distribución es un indicador de la variabilidad de la resistencia de los materiales, debido a una distribución de tamaños de las imperfecciones [22] y cuanto mayor es su valor, menor es la variabilidad de la propiedad medida. Las curvas se construyeron en el software OriginPro 8.5 y a partir de éste se obtuvo el módulo de Weibull, que representa el grado de variabilidad de cada una de las propiedades halladas. Además el valor de la dureza y del módulo de elasticidad fue hallado gráfi- 
camente como el percentil 50 .

\section{RESULTADOS Y ANÁLISIS}

\subsection{Morfología de la materia prima}

La morfología del polvo de circona estabilizada con $8 \%$ en mol de itria (8YSZ) fabricada por la casa comercial "US Research Nanomaterials" se muestra en la Figura 1. La micrografía muestra una magnificación de 30.000 aumentos, donde se aprecia que las partículas del polvo nanométrico se encuentran ligeramente aglomeradas y además se puede observar que el tamaño de las partículas del polvo 8YSZ está en un rango entre 100 y $200 \mathrm{~nm}$ aproximadamente.

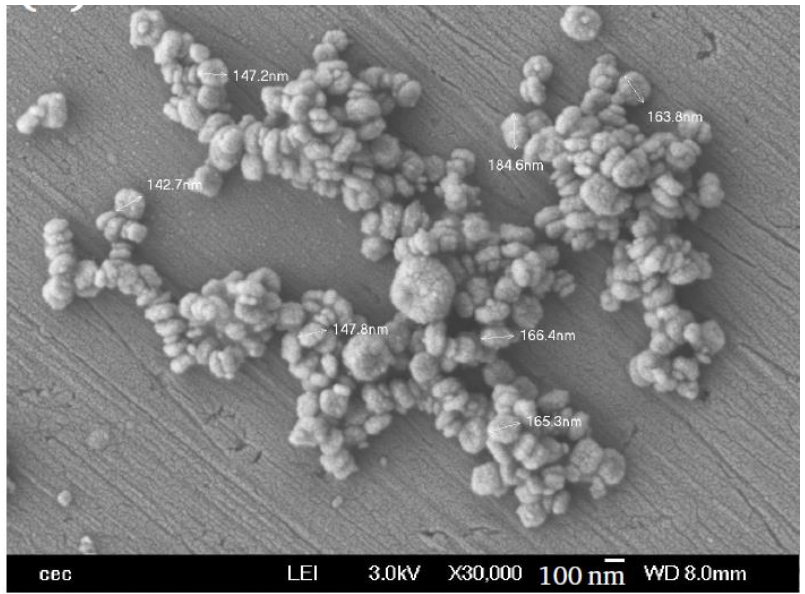

Figura 1: Morfología del polvo nanométrico de 8YSZ por FE-SEM (30000X)

\subsection{Análisis estructural del recubrimiento}

La sección transversal del recubrimiento 8YSZ elaborado por SPS se muestra en la Figura 2. Se observa que el recubrimiento presenta una estructura homogénea en general (Figura 2a), con presencia de poros y una baja densidad de grietas. Además el recubrimiento presenta un espesor de $83 \pm 2 \mu \mathrm{m}$ promedio de 5 medidas a 10 imágenes analizadas mediante el software ImageJ, según la norma ASTM B487-07 [23]. En la Figura 2b se muestra una ampliación del recubrimiento, el cual presenta una estructura compuesta por dos zonas: (i) una zona densa con partículas nanométricas semifundidas (ZS) y (ii) lamelas con mayor grado de fusión (ZF) que cubren la anterior zona.
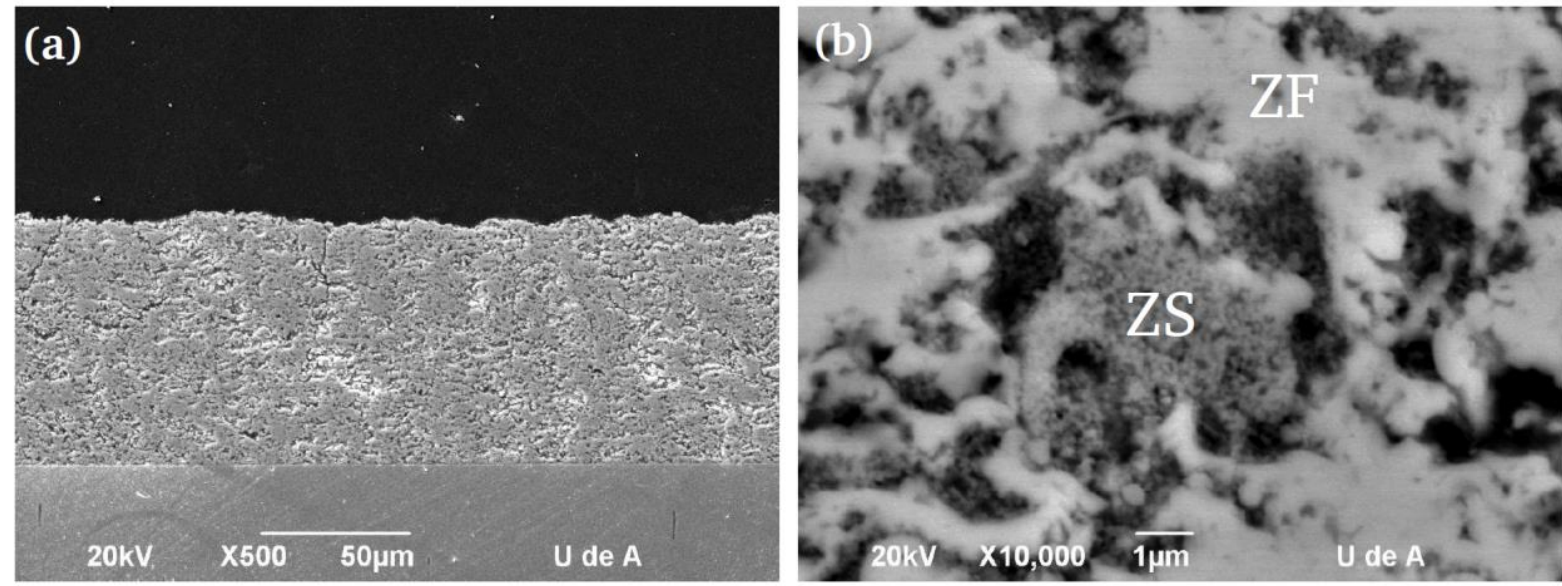

Figura 2: Sección transversal del recubrimiento 8YSZ (a) Vista de la interface recubrimiento - acero (b) Ampliación de la capa cerámica elaborada por SPS 
El resultado del análisis químico mediante FRX realizado al recubrimiento de 8 YSZ mostró los siguientes compuestos (\% en peso): $\mathrm{ZrO}_{2}$ con un $84.81 \%, \mathrm{Y}_{2} \mathrm{O}_{3}$ con un $12.48 \%, \mathrm{HfO}_{2}$ con un $1.54 \%, \mathrm{Al}_{2} \mathrm{O}_{3}$ con un $0.25 \%$ y otros con $0.92 \%$. Se puede observar que la composición química (\% en peso) analizado por FRX corresponden a la relación en mol entre la circona y la itria del recubrimiento de $8 \%$ en mol. El $\mathrm{HfO}_{2}$ es un compuesto que proviene del método de fabricación del polvo utilizado como materia prima.

\subsection{Caracterización mecánica del recubrimiento}

\subsubsection{Nanoindentación}

En la Figura 3 se muestra una micrografía con las huellas de nanoindentación, el patrón de la curva cargadescarga P-h y el modelo estadístico de Weibull para la dureza y el módulo de elasticidad. Las curvas P-h presentan un comportamiento típico de los materiales cerámicos, es decir, que la recuperación elástica es bastante notable [24]. Esto se observa en la disminución en la profundidad de todas las curvas de descarga, por ejemplo, en la curva dos de la Figura 3 b hubo una reducción en la profundidad de 1,2 a 0,95 $\mu$ m, lo cual incide en la forma de la huella residual e indica que la dureza del material no es uniforme y que depende de la zona indentada.
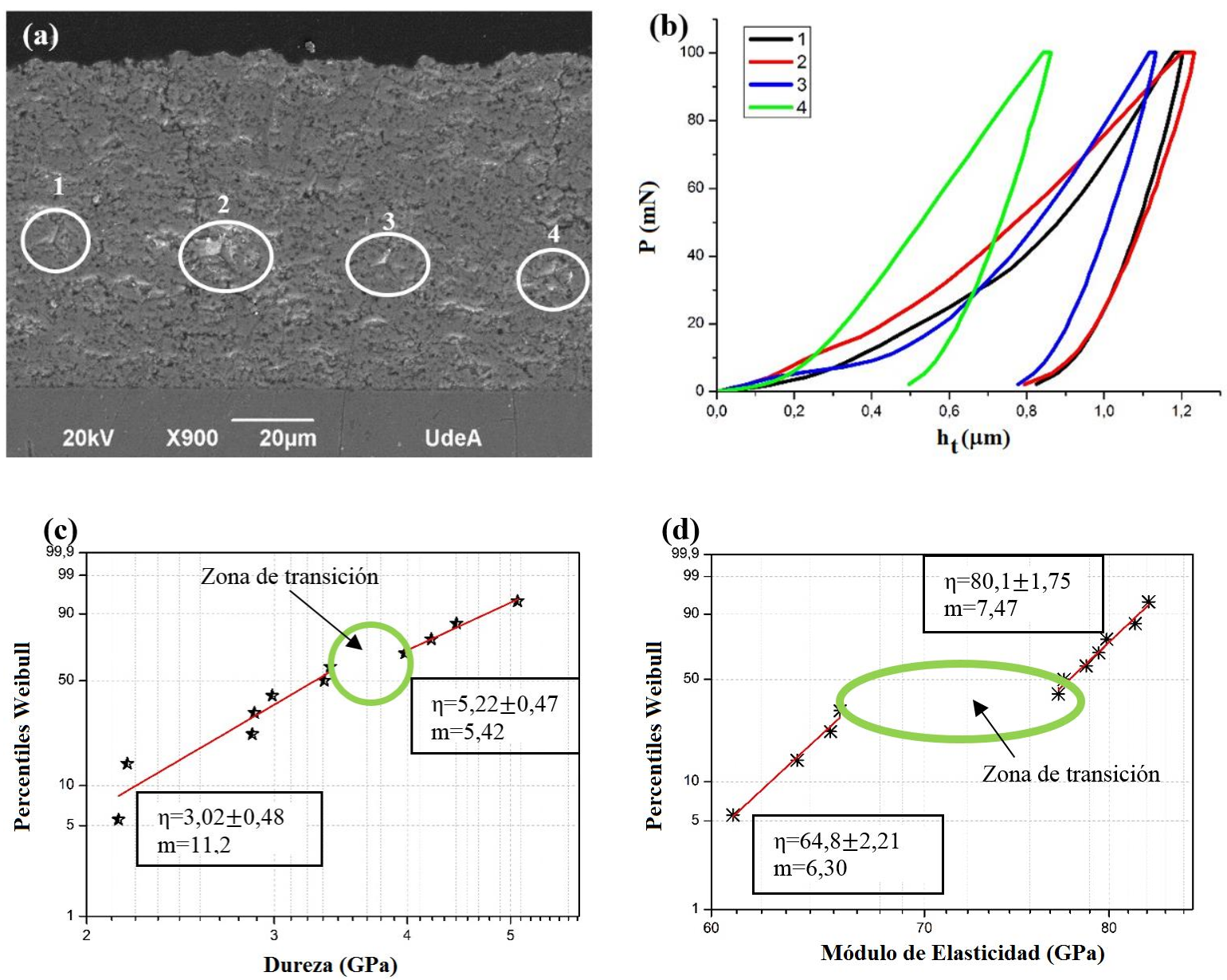

Figura 3: (a) Sección transversal del recubrimiento 8YSZ pulido con 4 huellas de nanoindentación realizadas. (b) Gráfico de la curva P-h de las cuatro nanoindentaciones de la micrografía. Modelo estadístico de Weibull para datos tomados de nanoindetación de (c) Dureza y (d) Módulo de elasticidad, donde $\eta$ es la dureza y módulo de elasticidad, respectivamente y m es el parámetro de forma del módulo de Weibull.

La Figura 3c muestra la gráfica de la distribución estadística de Weibull, en la cual se observa que el recubrimiento tiene una distribución bimodal, representada por dos zonas diferentes, una zona con partículas de mayor grado de fusión que tiene una dureza superior por el efecto de la sinterización [25] y otra zona con partículas menos fundidas que tienen dureza inferior. Además de las zonas mencionadas, se observa una 
tercera zona de transición, en la cual el valor de la dureza es intermedio. Se encontró que la zona más fundida (ZF) tiene una dureza de 5,22 $\pm 0,47 \mathrm{GPa}$ y que la zona menos fundida (ZS) tiene una dureza de 3,02 $\pm 0,48$ GPa. La dispersión de los datos se debe a la presencia de defectos en la vecindad de la indentación Berkovich. El módulo de elasticidad del recubrimiento 8YSZ medidos por nanoindentación también presenta una distribución bimodal, variando entre $80,1 \pm 1,75$ para ZF y $64,8 \pm 2,22$ GPa para ZS.

Tanto los valores de $\mathrm{H}$ como de E son inferiores a los reportados por la literatura para la zona más fundida para recubrimientos elaborados por SPS [9-11]. Esto puede deberse a que la ZF no está completamente fundida como se esperaba. La disminución en el grado de fusión puede atribuirse al corto tiempo de residencia de las partículas en el plasma y a que éstas no penetraron a la zona más caliente.

Los valores reportados por la literatura observados en trabajos como el de Carpio et al [1], encontró que hay tres tendencias claramente definidas en la profundidad de las curvas $\mathrm{H}$ y E. Un rango de dureza para la zona completamente fundida entre 8 y $12 \mathrm{GPa}$, una dureza en la zona semifundida que se estabilizó alrededor de $2 \mathrm{GPa}$ e indentaciones que alcanzaron la mezcla de estructuras mostrando valores de dureza de $4 \mathrm{GPa}$. Con respecto a los resultados del módulo de elasticidad, en el mismo trabajo [1] los autores reportan valores obtenidos que varían entre 125 y $150 \mathrm{GPa}$ para las zonas completamente fundidas y $65 \mathrm{GPa}$ para las semifundidas. Wang et al [13] encontró que los recubrimientos YSZ nanoestructurados obtenidos por APS también muestran una distribución bimodal de las propiedades mecánicas ( $\mathrm{H}$ y E), que correspondían a las zonas fundidas y parcialmente fundidas.

Por otro lado, los módulos de Weibull representados con "m” (ver Figura 3c y 3d) de la dureza y del módulo de elasticidad representan la variación de las propiedades medidas y presentan valores acordes a los encontrados en algunos estudios anteriores, como el de Wang et al [13], donde concluye que estos valores se deben a la heterogeneidad en la estructura de los recubrimientos y a la presencia de micro-defectos.

En la Figura 4 se muestra una imagen de dos huellas de nanoindentaciones realizadas en la sección transversal del recubrimiento. Se evidencia que existe una notable diferencia en el tamaño de la huella, que se ve reflejado en la dureza $(\eta)$ de las dos zonas que presenta el recubrimiento. La zona que tiene partículas nanométricas sin fundir (ver Figura 4a) es mucho más blanda que la zona con partículas de mayor grado de fusión (ver Figura 4b). Es importante resaltar que las dos indentaciones Berkovich fueron realizadas a las mismas condiciones.
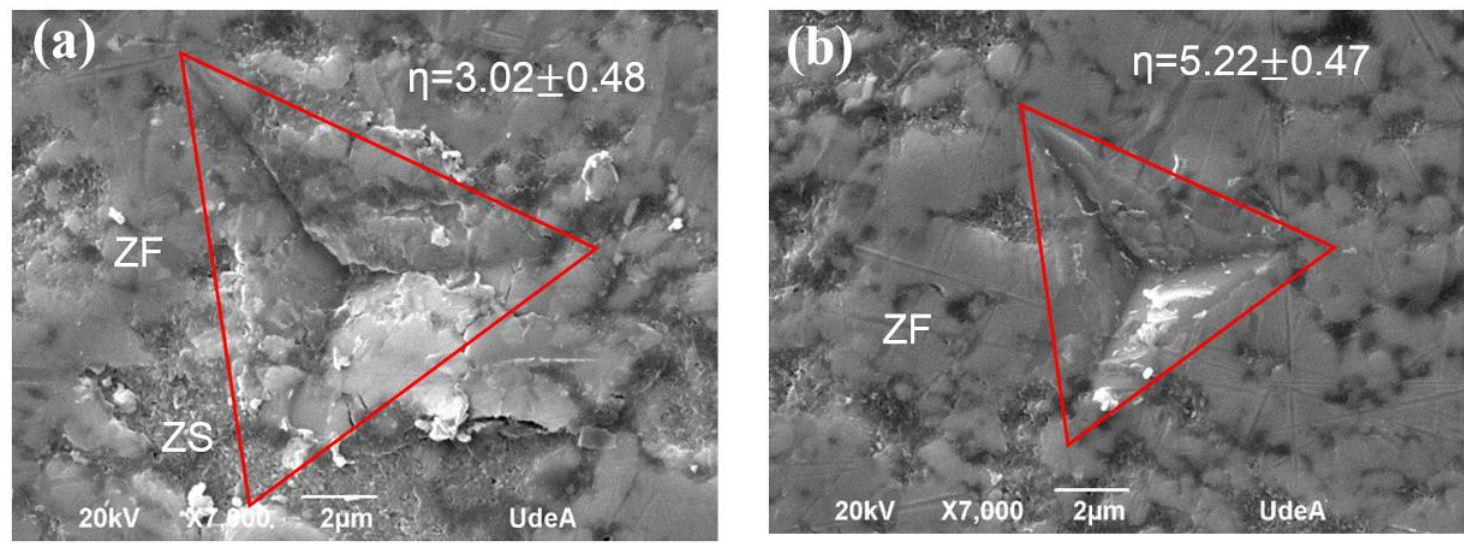

Figura 4: Huellas de nanoindentación realizadas en el recubrimiento 8YSZ en una (a) Zona con partículas nanométricas parcialmente fundidas (ZS) y (b) Zona de mayor grado de fusión (ZF). Las líneas rojas encierran las huellas de nanoindentación producidas por el indentador Berkovich.

\subsubsection{Microindentación}

La microdureza HV del recubrimiento fue hallada a partir de indentaciones realizadas con un indentador Vickers y el módulo de elasticidad fue calculado usando el método de Marshall [26], el cual se basa en el análisis de la huella residual producida con un indentador Knoop.

En los recubrimientos elaborados por SPS y en general los elaborados por proyección térmica, se generan inevitablemente defectos estructurales, tales como, partículas sin fundir, micro-grietas, poros abiertos o cerrados que hacen que las propiedades mecánicas de materiales con la misma composición química sean diferente [27]. Las huellas residuales producidas por microindentación en el recubrimiento fueron influenciadas por la presencia de micro-defectos que causaron una alta dispersión de los datos como en la mayoría de 
los recubrimientos elaborados por proyección térmica.

En la Figura 5 se observan las huellas de microindentación realizadas con un indentador Vickers y la curva del modelo estadístico de Weibull para la dureza y el módulo de elasticidad del recubrimiento 8YSZ. En la Figura 5b se observa que la región afectada por la indentación comprende las dos zonas del recubrimiento, lo que da un indicio que la indentación está midiendo las propiedades conjuntamente de las dos zonas. La curva Weibull de la microdureza HV (ver Figura 5b) muestra una distribución monomodal, lo cual está influenciado por la carga aplicada, la forma y el tamaño del indentador, ya que la región afectada de la indentación abarca ambas zonas (ZS y ZF), impidiendo discriminar entre una y otra. Sin embargo, se presentaron huellas que tomaron más cantidad de zonas que tenían más partículas semifundidas que otras, lo cual explica la gran desviación entre los datos. Se encontró que el recubrimiento tiene una dureza de $\mathrm{HV}=4,17$ $\pm 0,39 \mathrm{GPa}$ que corresponde al percentil 50 de la curva y con un módulo de Weibull $\mathrm{m}=12,2$. En la Figura $5 \mathrm{~d}$ se muestra la curva Weibull del módulo de elasticidad E y se observa un comportamiento monomodal con $\mathrm{E}=149 \pm 25 \mathrm{GPa}$ y un módulo de Weibull $\mathrm{m}=6,49$.
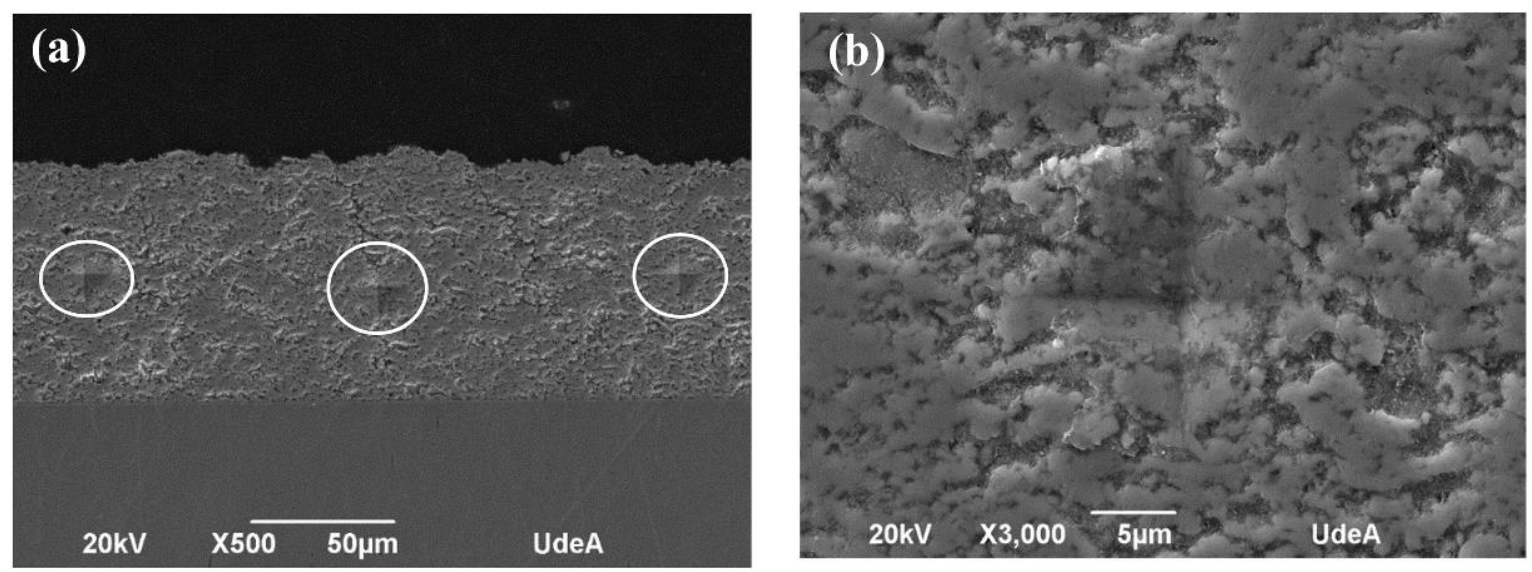

(c)
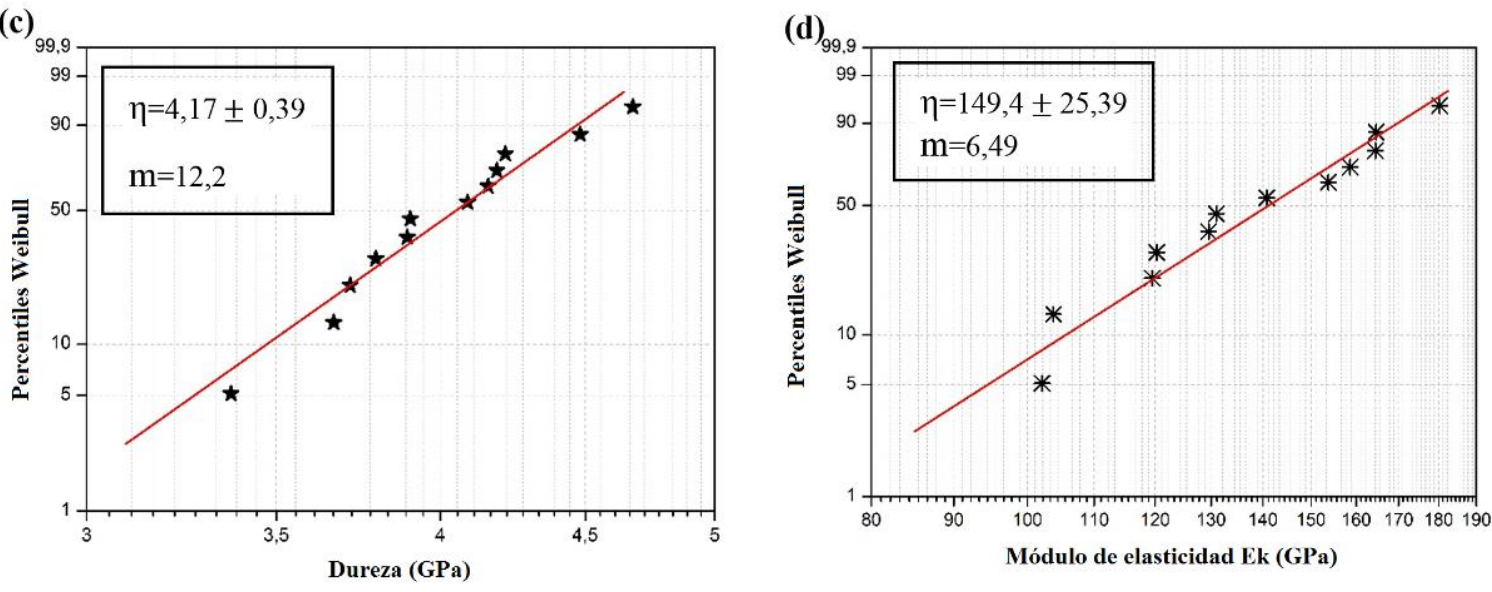

Figura 5: Sección transversal del recubrimiento 8YSZ, (a) Micrografía de las huellas de microindentación Vickers (b) Ampliación de una indentación representativa a 3000X, Modelo estadístico de Weibull para (c) Microdureza HV y el (d) Módulo de elasticidad E

En la literatura no se reportan trabajos de medida del módulo de elasticidad empleando el método de Marshall para recubrimientos elaborados por SPS, pero se encuentran algunos trabajos para recubrimiento elaborados por APS [28] que demuestran que este método es efectivo en la medida del módulo de elasticidad (E) de recubrimientos de YSZ empleados como barrera térmica. Por ejemplo, Singh [28] evaluó el módulo de elasticidad de recubrimientos de YSZ como barrera térmica, utilizando el método de Marshall, donde encontró un valor del módulo de elasticidad de $68,4 \pm 22,6 \mathrm{GPa}$ a una carga de indentación de $50 \mathrm{~g}$ y 35,7 \pm 9,8 GPa a una carga de indentación de 300 g. En este trabajo se resaltó que con cargas más altas, los valores del módulo elástico no cambiaron significativamente. 
Una consideración adicional que se debe tener en cuenta para entender la forma de las huellas obtenidas por microindentación en el tipo de recubrimiento estudiado (estructura bimodal), es el fenómeno de apilamiento que ocurre en la zona adyacente al área indentada. Cuando la zona adyacente al área indentada es más blanda (Zona semifundida, "ZS"), facilita la penetración del indentador, lo cual hace que las aristas de la huella obtenida adquieran una curvatura que le proporciona forma de barril tal y como se muestra en la Figura 6.

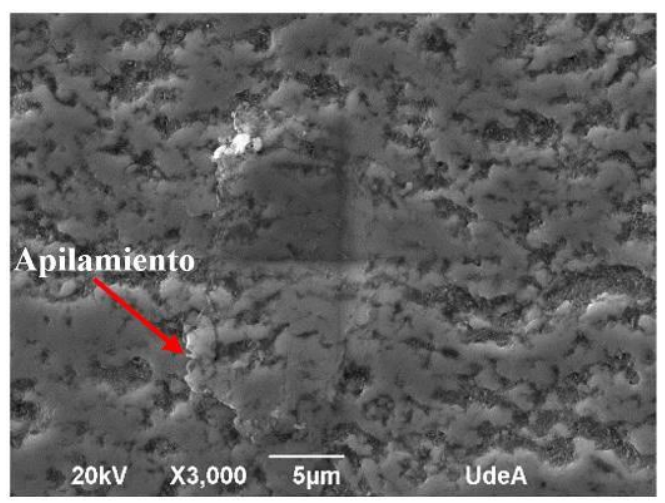

Figura 6: Huella de microindentación con efecto barril y desprendimiento del material del recubrimiento

\subsection{Comparación de las técnicas de indentación usadas}

Existen algunos factores ajenos al material que pueden condicionar el análisis de los datos experimentales. En particular, el efecto del tamaño de la indentación y la geometría del indentador empleado pueden producir variaciones considerables respecto a los resultados esperados. Sin embargo, dadas las múltiples aplicaciones que tiene el recubrimiento estudiado, no es posible concluir cuál de las técnicas empleadas es la más adecuada. No obstante, se pueden establecer varias consideraciones que dependiendo del tipo de aplicación y estudio, se necesitará preferentemente la información dada por una técnica u otra.

En la Tabla 1 se presenta un resumen de las durezas y los módulos de elasticidad medidas por nanoindentación y microindentación. Se observa que los valores de módulo de elasticidad hallados por microindentación presentan valores mayores comparados con los valores hallados por nanoindentación, ya que las huellas residuales en los ensayos de microindentación comprenden tanto la zona semifundida como la más fundida, tal como se observa en la Figura 7. Por otro lado, los ensayos de nanoindentación están en la capacidad de discriminar entre las diferentes regiones estructurales debido a que se utilizan cargas muy pequeñas, además de la forma del indentador. En la Tabla 1 también se observa que el valor de dureza medido por microindentación, presenta un valor intermedio entre los valores hallados por nanoindentación. Esto se explica por la distribución ecuánime entre las regiones con partículas nanométricas sin fundir (ZS) y las regiones más fundidas (ZF) (ver Figura 2) en el recubrimiento de 8YSZ, en el momento de realizar la microindentación.

Tabla 1: Resumen de las propiedades mecánicas

\begin{tabular}{c|c|c|c|c}
\hline \multirow{2}{*}{ Recubrimiento cerámico } & \multicolumn{2}{|c|}{$\begin{array}{c}\text { Nanoindentación } \\
\text { (GPa) }\end{array}$} & \multicolumn{2}{c}{$\begin{array}{c}\text { Microindentación } \\
\text { (GPa) }\end{array}$} \\
\cline { 2 - 5 } & Dureza & Módulo & Dureza HV & Módulo E \\
\hline \multirow{2}{*}{$8 Y S Z$} & & & & $149 \pm 25$ \\
& $3,02 \pm 0,48$ & $64,8 \pm 2,22$ & $4,17 \pm 0,39$ & \\
& $5,22 \pm 0,47$ & $80,1 \pm 1,75$ & & \\
\hline
\end{tabular}



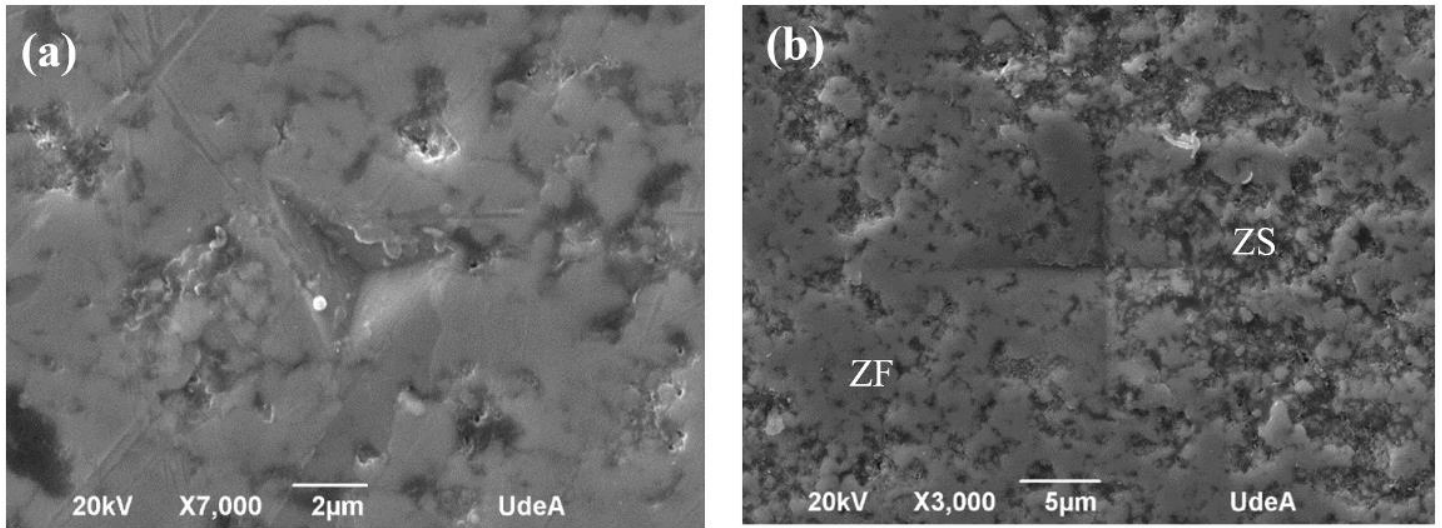

Figura 7: Huellas de (a) Nanoindentación y (b) Microindentación.

En la Tabla 2 se muestra un resumen de los módulos de Weibull del recubrimiento de 8YSZ. Esta distribución es un indicador de la variabilidad de las propiedades mecánicas de los materiales debido a una distribución de tamaños de las imperfecciones. Se observa que los valores del módulo de Weibull encontrados a partir de los resultados de las propiedades mecánicas halladas por microindentación y nanoindentación son similares. Esto indica que la variabilidad de las propiedades mecánicas halladas con ambas técnicas es muy equivalente. Esto se explica gracias a una distribución ecuánime entre las dos regiones (ZF y ZS) presentes en la estructura del recubrimiento (ver Figura 2b), lo cual permite que tanto las microindentaciones como las nanoindentaciones tengan probabilidades del mismo orden de magnitud de abarcar zonas del mismo comportamiento respecto a la propiedad medida (dureza y módulo de elasticidad). Es importante resaltar que ambas técnicas son sensibles a la presencia de defectos en el recubrimiento de 8YSZ, tales como poros y grietas como lo es reportado en el estudio de L. Wang [29].

Tabla 2: Resumen de los módulos de Weibull

\begin{tabular}{c|c|c|c|c}
\hline \multirow{2}{*}{$\begin{array}{c}\text { Recubrimiento } \\
\text { cerámico }\end{array}$} & \multicolumn{4}{|c}{ Módulo de Weibull } \\
\cline { 2 - 5 } & \multicolumn{2}{|c}{ Nanoindentación } & \multicolumn{2}{c}{ Microindentación } \\
\cline { 2 - 5 } & H & E & HV & E \\
\hline 8 YSZ & 11,2 & 6,30 & 12,2 & 6,49 \\
\hline
\end{tabular}

\section{CONCLUSIONES}

En este trabajo se estudió el efecto de la estructura en sus propiedades mecánicas medidas por nanoindentación y microindentación en un recubrimiento de 8 YSZ elaborado mediante la técnica de proyección térmica por plasma a partir de suspensiones (SPS) y cuyos análisis de resultados mostraron las siguientes conclusiones:

- El recubrimiento estudiado presentó una estructura de distribución bimodal, compuesta por (i) una zona densa con partículas nanométricas semifundidas (ZS) y (ii) lamelas con mayor grado de fusión que cubren la anterior zona $(\mathrm{ZF})$.

- Los datos de la medida de las propiedades mecánicas medidas por nanoindentación siguen una distribución de Weibull con las características bimodales y una dispersión debida a la hetereogeneidad y anisotropía de su estructura. Ademas, las regiones más fundidas (ZF) son claramente más rígidas y duras que las regiones menos fundidas (ZS) del recubrimiento de 8 YSZ elaborado por SPS.

- La nanoindentación representa una importante herramienta para la evaluación de las propiedades mecánicas en áreas específicas de recubrimientos con estructuras bimodales y aporta mucho en el desarrollo de recubrimientos fabricados por SPS.

- Se comprobó que la técnica de microindentación es adecuada para evaluar las propiedades mecánicas de recubrimientos fabricados por SPS de una manera más global, ya que los valores obtenidos en este estudio no permitieron discriminar las zonas nanoestructuradas (semifundidas, ZS) y las zonas 
completamente fundidas (ZF) del recubrimiento de 8YSZ.

- La diferencia entre el módulo de elasticidad hallado por nanoindentación y microindentación se debe a que ambas técnicas arrojaron información diferente, es decir, la técnica de nanoindentación por las bajas cargas aplicadas permite diferenciar entre las diferentes dos zonas estructurales del recubrimiento (ZS y $\mathrm{ZF}$ ) y en cambio la técnica de microindentación, permite profundidades y áreas de mayores penetración que en nanoindentación, debido a la diferencia del volumen, tipo de indentador y carga de la microindentación hace que se desplace las zonas rigidas (medidas por nanoindentación) incrementado en valor del modulo de elasticidad obtenido para el recubrimiento estudiado.

\section{AGRADECIMIENTOS}

Los autores agradecen a la Universidad de Antioquia (Colombia) por su financiación mediante los proyectos CODI MDC10-01-04, PR14-2-05 y al programa "Estrategia de Sostenibilidad 2014-2015 de la Universidad de Antioquia". El autor Andrés González agradece a Colciencias y Colfuturo por el apoyo financiero en sus estudios de doctorado realizados en la Universidad de Antioquia y en la Universidad de Limoges (Francia).

\section{BIBLIOGRAFIA}

[1] CARPIO, P., RAYÓN, E., PAWŁOWSKI, L. et al, "Microstructure and indentation mechanical properties of YSZ nanostructured coatings obtained by suspension plasma spraying", Surf. Coatings Technol., v. 220, pp. 237-243, Apr. 201.

[2] GONZÁLEZ, A. G., LÓPEZ, E., TAMAYO, A. et al, "Análisis de la microestructura y de las fases de recubrimientos de zirconia-alumina elaborados por proyección térmica," Dyna, v. 77, n. 162, pp. 151-160, 2009.

[3] GONZALEZ, A., HENAO, J., DIAZ, A. F., et al, "Influencia de los parámetros de proyección térmica en la microestructura de los recubrimientos de circona-alúmina y circona-ceria usados como barreras térmicas", Rev. Latinoam. Metal. y Mater., v. 33, n. 2, pp. 272-281, 2013.

[4] OSORIO, J. D., HERNÁNDEZ-ORTIZ, J. P., TORO, A. "Microstructure characterization of thermal barrier coating systems after controlled exposure to a high temperature", Ceram. Int., v. 40, n. 3, pp. 46634671, 2014.

[5] OSORIO, J. D., LOPERA-VALLE, A., TORO, A. et al, "Phase transformations in air plasma-sprayed yttria-stabilized zirconia thermal barrier coatings", Dyna, v. 81, n. 185, pp. 13-18, 2014.

[6] SILVA, M. A., BOAVENTURA, J. S., ALENCAR, M. G., et al, "Desenvolvimento de protótipo de células a combustível do tipo óxido sólido com reforma direta", Matéria, v. 12, n. 1, pp. 99-110, 2007.

[7] GONZÁLEZ, A., AGEORGES, H., ROJAS, O., et al, "Efecto de la microestructura y de la microdureza sobre la resistencia al desgaste de recubrimientos elaborados por proyección térmica por plasma atmosférico a partir de circona-alúmina, circona-itria y circona-ceria", Bol. la Soc. Esp. Ceram. y Vidr., v. 54, n. 3, pp. 124-132, 2015.

[8] FAUCHAIS, P., RAT, V., COUDERT, J. F., et al, "Operating parameters for suspension and solution plasma-spray coatings", Surf. Coatings Technol., v. 202, n. 18, pp. 4309-4317, 2008.

[9] GHASEMI, R., SHOJA-RAZAVI, R., MOZAFARINIA, R., et al., "Comparison of microstructure and mechanical properties of plasma-sprayed nanostructured and conventional yttria stabilized zirconia thermal barrier coatings", Ceram. Int., v. 39, n. 8, pp. 8805-8813, Dec. 2013.

[10] VANEVERY, K., KRANE, M. J. M., TRICE, R. W. "Parametric study of suspension plasma spray processing parameters on coating microstructures manufactured from nanoscale yttria-stabilized zirconia", Surf. Coatings Technol., v. 206, n. 2012, pp. 2464-2473, 2012.

[11] GONZÁLEZ HERNÁNDEZ, A. G. Estudio del comportamiento a alta temperatura de recubrimientos nanoestructurados elaborados por proyección térmica por combustión y plasma a partir de polvos y suspensiones, Tesis de Doctorado., Universidad de Antioquia, 2014.

[12] PAWLOWSKI, L. "Suspension and solution thermal spray coatings", Surf. Coatings Technol., v. 203, no. 19, pp. 2807-2829, 2009.

[13] WANG, L., ZHONG, X. H., YANG, J. S., et al, "Finite element simulation of surface micro-indentation behavior of yttria stabilized zirconia thermal barrier coatings with microstructural characteristic of columnar grains and sub-grains based on a nonlinear contact model”, Comput. Mater. Sci., v. 82, pp. 244-256, Feb. 2014. 
[14] KOZERSKI, S., LATKA, L., PAWLOWSKI, L., et al, "Preliminary study on suspension plasma sprayed $\mathrm{ZrO}_{2}+8$ wt.\% $\mathrm{Y}_{2} \mathrm{O}_{3}$ coatings", J. Eur. Ceram. Soc., v. 31, no. 12, pp. 2089-2098, Oct. 2011.

[15] CARPIO, P., BANNIER, E., SALVADOR, M. D., et al, et al, "Effect of particle size distribution of suspension feedstock on the microstructure and mechanical properties of suspension plasma spraying YSZ coatings", Surf. Coatings Technol., Sep. 2014.

[16] ETCHART-SALAS, R., "Projection par plasma d'arc de particules submicroniques en suspension. Approche expérimentale et analytique des phénomènes impliqués dans la reproductibilité et la qualité des dépôts", Tesis de Doctorado, Université de Limoges, 2007.

[17] BACCIOCHINI, A., "Quantification de l'architecture poreuse de dépôts finement structurés (submicromètre - nanomètre) de zircone yttriée realisés par projection plasma de suspension", Tesis de Doctorado, Université de Limoges, 2010.

[18] ASTM E1920-03, "Standard Guide for Metallographic Preparation of Thermal Sprayed Coatings," 2003.

[19] OLIVER, W.C., PHARR, G.M. "An improved technique for determining hardness and elastic modulus using load and displacement sensing indentation experiments", J. Mater. Res., v. 7, pp. 1564-1583, 1992.

[20] ASTM E1327-08, "Standard Test Method for Vickers Indentation Hardness of Advanced Ceramics," 2008.

[21] ASTM E1326-08, "Standard Test Method for Knoop Indentation Hardness of Advanced Ceramics," 2003.

[22] ASKELAND, D. R., PHULÉ, P. P. "Propiedades y comportamiento mecánico",In: Ciencia e ingeniería de los materiales, 4 ed., p. 278, 2003.

[23] ASTM B487-85, "Standard Test Method for Measurement of Metal and Oxide Coating Thickness by Microscopical Examination of Cross Section 1," Annu. B. ASTM Stand., v. i, pp. 1-4, 2011.

[24] ASTRID, M. "Caracterización de las propiedades tribológicas de los recubrimientos duros," Tesis de Doctorado, Universidad de Barcelona, 2005.

[25] RODRÍGUEZ, D. M. "Correlación de la microestructura con la tenacidad a la fractura en recubrimientos de barrera térmica (TBC) para aplicaciones de turbinas a gas", Tesis de Maestría, Universidad Nacional de Colombia, 2013.

[26] MARSHALL, D. B., NOMA, T., EVANS, A.G."A Simple Method for Determining Elastic-Modulus-toHardness Ratio using Knoop Indentation Measurements”, J. Am. Ceram. Soc., v. 65, pp. 175-176, 1982.

[27] KANG, J., MA, J., LI, G., et al, "Bimodal distribution characteristic of microstructure and mechanical properties of nanostructured composite ceramic coatings prepared by supersonic plasma spraying", Mater. Des., v. 64, pp. 755-759, 2014.

[28] SINGH, J. P., SUTARIA, M., M. FERBER, "Use of indentation technique to measure elastic modulus of plasma-sprayed zirconia thermal barrier coatings", Ceram. Eng. Sci. Proc., pp. 191-200, 1997.

[29] WANG, L., WANG, Y., SUN, X. G. "Microstructure and indentation mechanical properties of plasma sprayed nano-bimodal and conventional $\mathrm{ZrO}_{2}-8 \mathrm{wt} \% \mathrm{Y}_{2} \mathrm{O}_{3}$ thermal barrier coatings", Vacuum, v. 86, n. 8, pp. 1174-1185, Feb. 2012. 